\title{
Adaptive Hexapod Simulator Motion based on Aircraft Stability
}

\author{
Peter M. T. Zaal* \\ San José State University \\ NASA Ames Research Center \\ Moffett Field, CA, 94035
}

\author{
Alexandru Popovici ${ }^{\dagger}$ \\ San José State University \\ NASA Ames Research Center \\ Moffett Field, CA, 94035
}

\author{
Emily K. Lewis \\ Metis Technology Solutions \\ NASA Ames Research Center \\ Moffett Field, CA, 94035
}

This paper determined the feasibility of an adaptive hexapod simulator motion algorithm based on aircraft roll stability. An experiment was conducted that used a transport aircraft model in the Vertical Motion Simulator at NASA Ames Research Center. Eighteen general aviation pilots flew a heading-capture task and a stall task consecutively under four motion configurations: baseline hexapod, adaptive hexapod, optimized hexapod, and full motion. The adaptive motion was more similar to the baseline hexapod motion in the heading-capture task when the aircraft was more stable, and more similar to the optimized hexapod motion in the stall task when the aircraft was more unstable. Pilot motion ratings and task performance in the heading-capture task under the adaptive hexapod motion were more similar to baseline hexapod motion compared to optimized hexapod motion. However, motion ratings and task performance in the stall task under the adaptive motion were not significantly more similar to the optimized hexapod motion compared to baseline hexapod motion. Motion ratings and overall task performance under optimized hexapod motion as opposed to baseline hexapod motion were always more similar to the full motion condition. This paper showed that adaptive motion based on aircraft stability is feasible and can be implemented in a straightforward way. More research is required to test the adaptive motion algorithm in different tasks.

\section{Nomenclature}

$\begin{array}{lll}b & = & \text { wing span, } \mathrm{ft} \\ C_{l} & = & \text { rolling moment coefficient, }- \\ C_{l_{0}} & = & \text { static rolling moment coefficient, }- \\ C_{l_{\delta a}} & = & \text { derivative of the rolling moment with } \\ & & \text { respect to aileron deflection angle, } \mathrm{rad}^{-1} \\ C_{l_{\beta}} & = & \text { derivative of the rolling moment with } \\ & & \text { respect to sideslip angle, } \mathrm{rad}^{-1} \\ C_{l_{p}} & = & \text { roll damping coefficient, } \mathrm{rad}^{-1} \\ C_{l_{r}} & = & \text { derivative of the rolling moment with } \\ & & \text { respect to yaw rate, rad } \\ d f & = & \text { statistical-test degree of freedom } \\ \bar{e}_{\psi} & = & \text { average heading error, deg } \\ H & = & \text { measurement vector } \\ H_{m} & = & \text { motion washout filter } \\ h & = & \text { altitude, } \mathrm{ft} \\ I_{s} & = & \text { stall alarm activation } \\ K & = & \text { Kalman gain, }- \\ K_{m} & = & \text { motion filter gain, }-- \\ K_{t} & = & \text { center of gravity translational } \\ & & \text { acceleration gain, }-\end{array}$

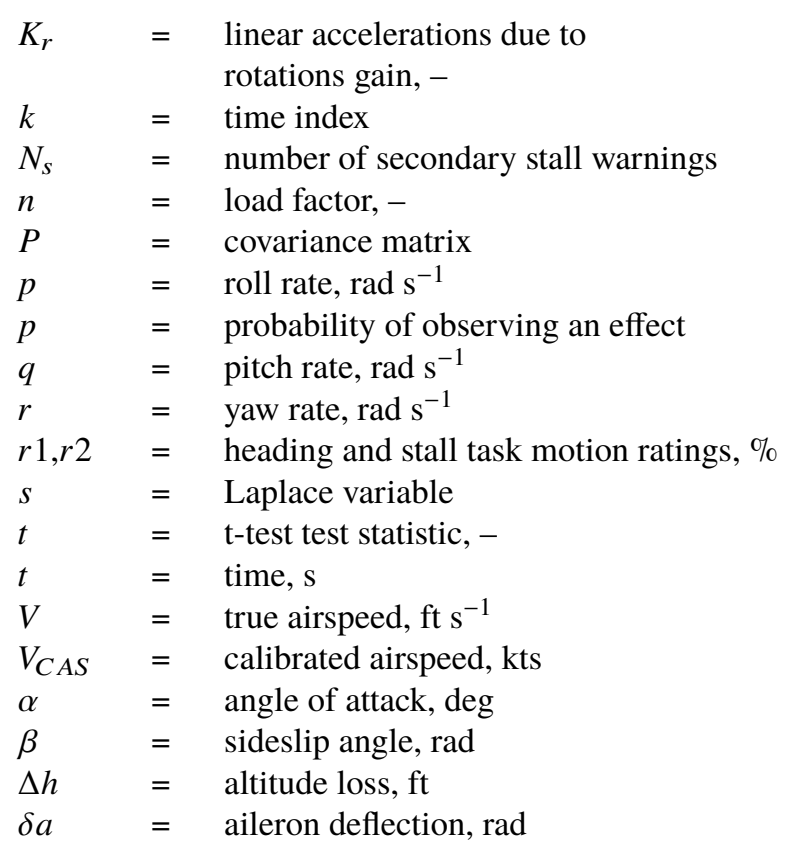

*Senior Research Engineer, Human Systems Integration Division, NASA Ames Research Center, Moffett Field, CA, 94035; peter.m.t.zaal@nasa.gov. Senior Member AIAA.

${ }^{\dagger}$ Research Associate, Human Systems Integration Division, NASA Ames Research Center, Moffett Field, CA, 94035; alexandru.popovici@ nasa.gov. Member.

${ }^{\ddagger}$ Principal Simulation Engineer, SimLabs, NASA Ames Research Center, Moffett Field, CA, 94035; emily.k.lewis@ nasa.gov. 


$\begin{array}{lll}\zeta_{m} & =\text { motion filter damping ratio, }- \\ \theta & = & \text { aircraft pitch angle, deg } \\ \theta & = & \text { parameter vector } \\ \lambda & = & \text { forgetting factor, }-\end{array}$

$\begin{array}{lll}\phi & = & \text { aircraft roll angle, deg } \\ \psi & = & \text { aircraft yaw angle, deg } \\ \omega_{m} & = & \text { motion filter break frequency, rad s }\end{array}$

\section{Introduction}

THIS paper presents a study conducted in the Vertical Motion Simulator (VMS) at NASA Ames Research Center to develop and evaluate an adaptive motion cueing algorithm based on aircraft roll stability. Airline pilots are required to perform full stall recovery training in flight simulators [1]. Historically, training simulators weren't set up to provide training at conditions outside their normal flight envelope, such as at angles of attack above the stall warning threshold. Post-stall aircraft models are required to simulate the aircraft response after the stall point [2, 3]. In addition, motion cues need to adequately represent this response to ensure the skills learned in simulator training are directly usable in real flight [4].

Under NASA's Airspace Operations and Safety Program, the Technologies for Airplane State Awareness (TASA) subproject conducts research to support the Commercial Aviation Safety Team (CAST) Safety Enhancement 209 (SE209) to study simulator fidelity improvements for commercial aircraft stall training [5]. Under this SE209 research, four simulator studies with pilots were conducted in the Vertical Motion Simulator (VMS) at NASA Ames Research Center, to develop a hexapod motion cueing strategy for stall recovery training in commercial transport simulators [4, 6-8]. An additional study in a level-D-certified B747 full flight simulator verified the motion cueing strategy with commercial airline pilots [9]. This motion cueing strategy prioritizes translational accelerations as a result from rotating around the aircraft's center of gravity over translational accelerations of the center of gravity, allowing for a higher fidelity of the motion cues that directly help a pilot damp the flight path response in a stall recovery, as well as stabilize the progressively less-stable roll dynamics and roll off near stall.

The developed motion cueing strategy, and others in the past [10], improve motion cues for stall recovery training; however, these motion cues might not be optimal for flight tasks in the normal flight envelope where the aircraft is stable. Previous work switched instantaneously between motion settings optimized for normal flight and upset recovery using predefined flight variable limits [11]. Adaptive motion cueing algorithms for upset recovery training allow for a more gradual change in motion cueing, but the parameters of previously developed algorithms varied based on the location of the simulator cab in the motion envelope [12]. The change in aircraft stability between the normal flight and stall regimes results in a change of usefulness of different motion cues and would therefore be a more logical driver for adaptive motion. This paper is the first to develop an adaptive hexapod motion algorithm based on aircraft roll stability. The adaptive motion algorithm was evaluated in an experiment on the VMS using 18 general aviation pilots who performed tasks in the normal and stall regimes of the flight envelope consecutively. Pilot motion ratings and performance with the adaptive motion were evaluated against baseline and optimized hexapod motion, and full motion more similar to that experienced in a real aircraft.

The paper is structured as follows. The flight task and adaptive motion are described in Section III, which is followed by a description of the experiment setup in Section IV. The results of the experiment are provided in Section V and discussed in Section VI. Section VII provides an overview of the main conclusions of this paper.

\section{Flight Tasks}

The goal of this study was to investigate the feasibility of adaptive simulator motion based on the roll stability of aircraft dynamics. To determine the effects of the adaptive motion on pilots' motion perception and task performance, a simulation scenario was designed which encompassed tasks in both the stable and unstable regimes of the flight envelope.

The scenario started during cruise at $190 \mathrm{kts}$ and an altitude of 40,000 ft. To increase pilots' task load, moderate turbulence was present throughout the scenario, generated by two sums-of-sines signals added to the aircraft roll and pitch attitudes, respectively [8]. In addition, the scenario was performed in the clouds; that is, without any visual attitude references from the out-the-window view. Pilots first performed two heading changes (task 1). The first heading change from 300 to $280 \mathrm{deg}$ started at $t=20 \mathrm{~s}$. The second heading change from 280 to $300 \mathrm{deg}$ started at $t=55 \mathrm{~s}$. Pilots were instructed to bank left or right to approximately $15 \mathrm{deg}$ and maintain a pitch attitude of approximately 8 deg while changing their heading. A tone at $t=20$ and $t=55$ signalled the start of the heading changes. Roll, pitch, and heading bugs marked the target roll and pitch attitudes, and desired heading on the primary flight display (PDF), respectively. 
After the last heading change, pilots had to initiate and recover from a high-altitude stall starting at $t=90 \mathrm{~s}$ (task 2). Pilots were instructed to increase their pitch angle to 12 degrees and maintain wings level as indicated by a flight director. The throttles were automatically back-driven to idle starting at $t=90 \mathrm{~s}$ as well. A stall-warning alarm sounded when the red minimum speed barber pole hit the current airspeed on the PDF. At $t=140 \mathrm{~s}$, once a negative climb rate was achieved, indicating a stall, the flight director disappeared and pilots had to recover from the stall. Pilots were not to recover until the flight director disappeared. The stall recovery was not guided by any display indicators. The correct recovery procedure was to lower the nose to approximately $-10 \mathrm{deg}$ pitch attitude, level the wings, and apply full throttle. Pilots were instructed to slowly pull up after an airspeed above $190 \mathrm{kts}$ was reached to achieve a positive rate of climb. They were encouraged to not activate any additional stall alarms during the recovery. The simulation scenario terminated after $200 \mathrm{~s}$.

Note that every task segment was triggered by time, not pilot performance, in order to create a scenario that could be repeated consistently between runs and pilots. All timings were determined during test runs and were selected to provide enough time to complete each task segment.

Example recordings for aircraft altitude, roll, pitch, heading, angle of attack, calibrated airspeed, load factor, and stall alarm activation during one run of the simulation scenario are provided in Fig. 1. Gray vertical lines separate the different segments of the tasks. Black dashed lines indicate the roll, pitch, and heading reference angles. During the heading changes, all aircraft parameters remained approximately constant except for the bank angle and heading. During the approach to stall, the altitude increased, airspeed decreased, and angle of attack increased gradually. Note that in these specific flight conditions, the stall alarm activated around an angle of attack of 15 deg. No secondary stall-alarm activations were observed in the stall recovery.

\section{A. Aircraft Model}

The General Transport Model (GTM) was used to simulate the aircraft dynamics. This full-scale simulation model is representative of a generic aircraft similar to a Boeing 757, and includes accurate post-stall dynamics [13-15]. This model was developed from a sub-scale polynomial aerodynamic database, extended to cover the stall regime with wind-tunnel and spin-tunnel test data. The model was further adapted to represent a full-scale aircraft by making Reynolds Number corrections. The model used a basic yaw damper. All other stability and control augmentation functions were switched off. The simulation model was implemented in the FLTz simulation environment [16]. At the start of each run the aircraft was trimmed at an altitude of 40,000 ft and an indicated airspeed of $190 \mathrm{kts}$. The gross weight of the airplane was $185,000 \mathrm{lbs}$.

\section{B. Roll Damping Coefficient}

In an approach to stall, the roll damping coefficient of the airplane decreases, resulting in unstable roll behavior $[9,14]$. This parameter is therefore a good indicator for the aircraft's transition from stable to unstable flight, a regime where different motion cues become more important for human manual control [4].

Since the GTM model did not provide a direct output of the roll damping coefficient, this parameter had to be identified in real time. The roll damping coefficient can be obtained from the aircraft's rolling moment coefficient, given in Eq. (1).

$$
C_{l}=C_{l_{0}}+C_{l_{\beta}} \beta+C_{l_{p}} \frac{p b}{2 V}+C_{l_{r}} \frac{r b}{2 V}+C_{l_{\delta a}} \delta a
$$

where $C_{l}$ is the rolling moment coefficient available from the GTM model, $p$ the roll rate, $r$ the yaw rate, $b$ the wing span, $V$ the true airspeed and $\delta a$ the the airplane's aileron deflection. Since rudder control was disabled during the task, the effect of the rudder deflection was omitted in Eq. (1). The roll damping coefficient is related to the value of $C_{l_{p}}$. The lower the magnitude of $C_{l_{p}}$, the lower the roll damping coefficient. All 5 coefficients in Eq. (1) were

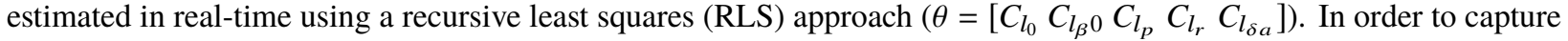
the time-varying characteristics of $C_{l_{p}}$, the covariance matrix of all parameters was increased every 20 seconds. This increased the uncertainty of the parameter estimation periodically. A summary of the RLS algorithm used is shown in Algorithm 1. 

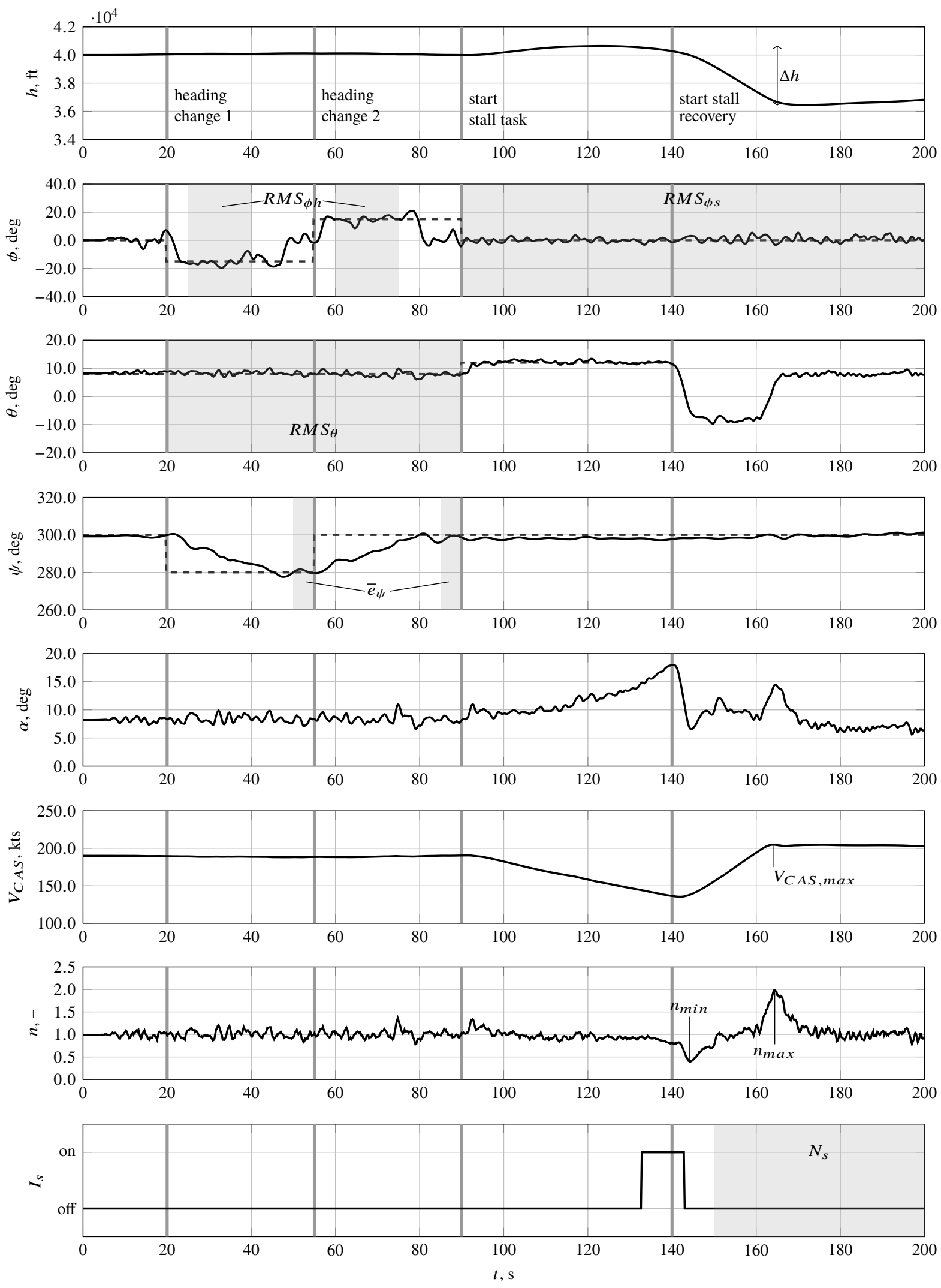

Fig. 1 Flight variable recordings. 


$$
\begin{aligned}
& \mathrm{P}(1)=\operatorname{diag}\left[\begin{array}{lllll}
0.1 & 0.1 & 1 & 0.1 & 0.1
\end{array}\right] ; \\
& \theta(1)=\left[\begin{array}{lllll}
0.0 & 0.0 & -0.3 & 0.0 & 0.0
\end{array}\right]^{T} ; \\
& \text { for } k=2: N \text { do } \\
& \mathrm{y}(\mathrm{k})=C_{l}(\mathrm{k}) \\
& \mathrm{H}=\left[\begin{array}{lllll}
1 & \beta(k) & \frac{p(k) b}{2 v(k)} & \frac{r(k) b}{2 v(k)} & \delta a(k)
\end{array}\right] ; \\
& \mathrm{K}=\frac{P(k-1) H^{T}}{\lambda+H P(k-1) H^{T}} ; \\
& \theta(\mathrm{k})=\theta(\mathrm{k}-1)+\mathrm{K}(\mathrm{y}(\mathrm{k})-\mathrm{H} \theta(\mathrm{k}-1)) ; \\
& \mathrm{P}(\mathrm{k})=\mathrm{P}(\mathrm{k}-1)(\mathrm{I}-\mathrm{KH}) \text {; }
\end{aligned}
$$

Algorithm 1: Recursive Least Squares algorithm.

In Algorithm 1, $P$ represents the state covariance matrix, $\theta$ the parameter vector, $H$ the measurement vector and $K$ the Kalman gain used in the RLS algorithm. In preliminary testing, $C_{l_{p}}$ was found to be, on average, around -0.30 in stable flight, and -0.15 during the more unstable stall task, just before the initiation of the stall recovery. The forgetting factor $\lambda$ was set to a small value, 0.001 , tuned for high responsiveness of the parameter estimation, which was particularly important during the approach to stall.

Fig. 2 provides an example of the estimate of $C_{l_{p}}$ corresponding to the data presented in Fig. 1. During the heading changes, until $t=90 \mathrm{~s}$, the roll damping coefficient remained around -0.30 . The aircraft became more unstable during the approach to the stall with $C_{l_{p}}$ increasing to -0.15 . Finally, $C_{l_{p}}$ decreased again during the stall recovery.

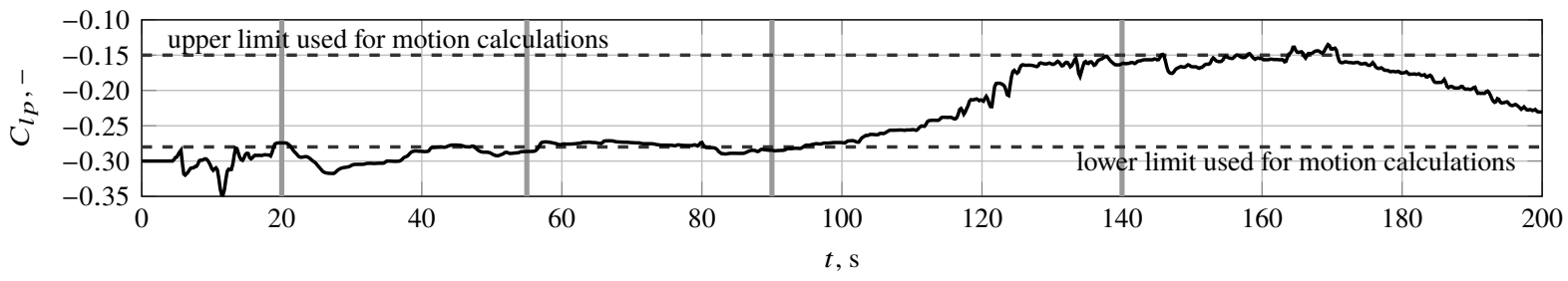

Fig. 2 Roll damping coefficient recording.

\section{Simulator Motion}

The experiment had a total of four different motion conditions: a baseline hexapod, optimized hexapod, adaptive hexapod, and full motion condition. The full motion condition utilized as much of the VMS motion space as possible, creating a so-called truth motion case, which likely represents the best that a ground-based simulator can do. The hexapod motion conditions simulated a six-degree-of-freedom hexapod motion platform, similar to ones used on current Level-D training simulators.

Fig. 3 depicts the overall motion logic. The standard VMS motion algorithm controlled the full motion condition. Some details are provided in [17]. The hexapod motion conditions utilized a motion algorithm developed by Parrish et al. [18]. The adaptive part of the algorithm was significantly modified from using platform motion constraints to using $C_{l_{p}}$ to drive the change in motion filter parameters. For the baseline and optimized hexapod motion configurations, the adaptive part of the algorithm was turned off. The algorithm calculated the leg extensions of the hexapod, thus accounting for the inherent constraints when trying to simultaneously move the hexapod in several degrees of freedom. Since the VMS has independent degrees of freedom, the resulting position and orientation commands after accounting for a possible leg reaching its limit had to be calculated. A Newton-Raphson optimization took the leg extensions and solved for the platform position and orientation in real-time. Next, the motion platform positions, rates and accelerations were sent to the VMS motion system (Fig. 3). The maximum extension of the hexapod legs was 60 inches. Mass and inertia effects of the hexapod system were not taken into account. As such, identical hardware dynamics resulted for each motion condition. The equivalent time delays of the VMS motion system for the pitch, roll, yaw, longitudinal, lateral, and vertical axes are, 47, 68, 48, 50, 69, and $67 \mathrm{~ms}$, respectively [17]. Both the VMS and hexapod motion 


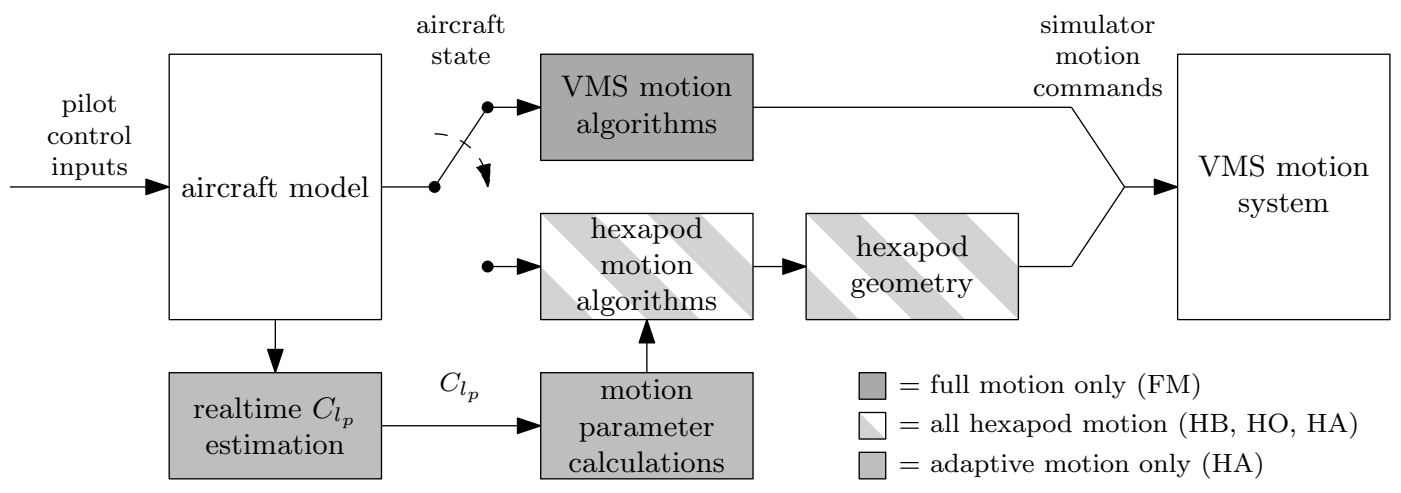

Fig. 3 Motion logic.

algorithms used second-order high-pass filters to attenuate the translational and rotational accelerations.

The baseline hexapod motion condition used motion filter parameter settings found in the average training simulator. The motion parameters for this condition were determined using the mean motion response of a statistical sample of eight representative hexapod motion simulators $[19,20]$. The optimized hexapod condition prioritized translational accelerations as a result of rotations with respect to the center of gravity over translational accelerations of the center of gravity allowing for significantly higher gains and lower break frequencies compared to the baseline hexapod motion condition $[4,9]$.

The adaptive hexapod motion varied between the baseline and optimized hexapod motion parameter settings based on the value of $C_{l_{p}}$. Only the motion filter gains and break frequencies were adaptive. The damping ratios were constant with a value of 0.707 . For $C_{l_{p}}<=-0.28$, the values of the motion filter gains and break frequencies were equal to those of the baseline hexapod configuration. The parameters were equal to those of the optimized hexapod configuration for $-0.15<=C_{l_{p}}$. The motion parameter values varied linearly with $C_{l_{p}}$ for $-0.28<C_{l_{p}}<-0.15$. Note that the variations in $C_{l_{p}}$ indirectly depend on pilots' control inputs and may be different for every run. Hence, the changes in simulator motion parameter values might also be different from run to run. An example of the motion parameter variations for the adaptive hexapod motion condition is provided in Fig. 4. Data in this figure correspond to the data in Figs. 1 and 2.

More details about the motion filters are provided in the appendix. Table 2 in the appendix summarizes the motion parameter settings for all four motion conditions. This table also contains the labels for each motion condition used throughout the remainder of the paper.
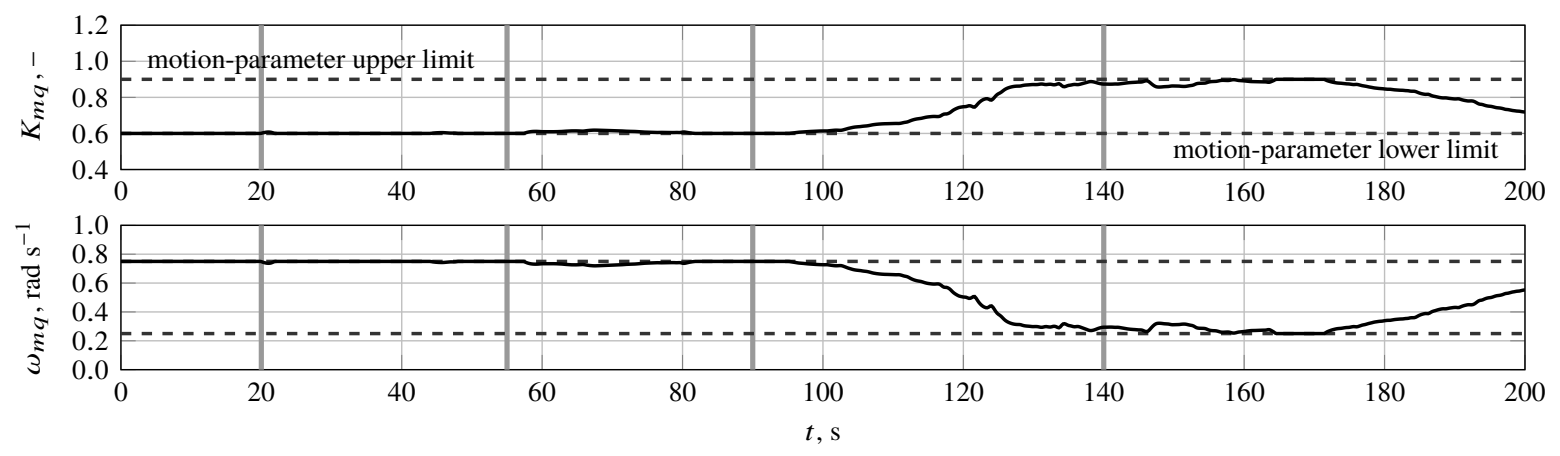

Fig. 4 Adaptive pitch motion filter parameters. 


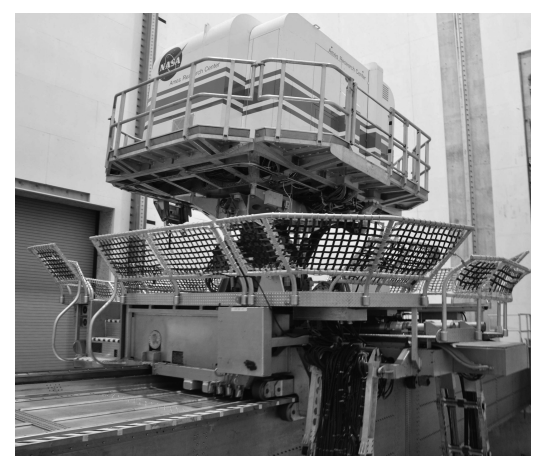

Fig. 5 Vertical Motion Simulator.

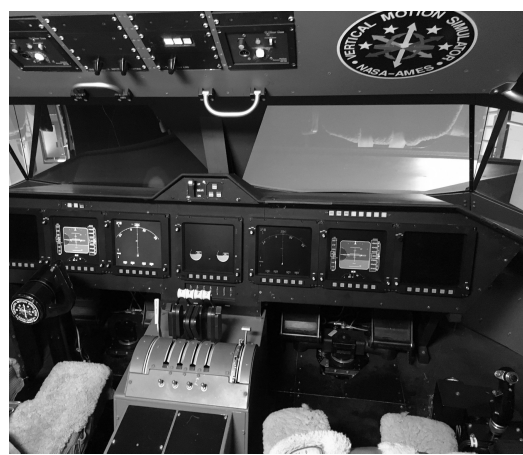

Fig. 6 Cockpit setup.

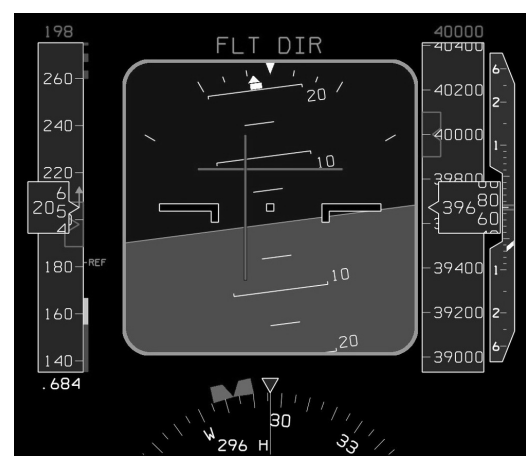

Fig. 7 Primary flight display.

\section{Experiment Setup}

\section{A. Method}

\section{Independent Variable}

The experiment had a within-subjects design with one independent variable: motion configuration with four levels (baseline hexapod motion (HB), optimized hexapod motion (HO), adaptive hexapod motion (HA), and full VMS motion (FM). In the HA condition, the motion tuning changed from $\mathrm{HB}$ to $\mathrm{HO}$ according to the aircraft's roll damping coefficient $C_{l_{p}}$. That is, in the heading changes task, the motion was similar to the HB condition, and in the stall and recovery tasks, the motion became similar to the HO motion condition.

\section{Apparatus}

The experiment was conducted in the VMS with the transport aircraft cab (T-cab), see Fig. 5. This cab has two seats. The left seat had a wheel and column to make control inputs. Participants performed the experiment from the right seat, which had a sidestick on the right side to make control inputs. Rudder pedals were available for both seats, however these were not operational during the experiment. Throttle levers were located in between the seats. The throttles were in auto-throttle mode and were automatically backdriven without pilot inputs for part of the simulation scenario (Section III). A PFD with representative V-speed markings, a navigation display, and an engine display were located in front of both seats (Fig. 6).

The out-the-window visual cues of T-CAB were collimated and provided by a system that projected a high-quality image on six spherical mirrors. The mirrors formed a dome-like section providing a continuous field-of-view image to both pilots. The out-the-window visual had a $220^{\circ}$ horizontal field of view and a $28^{\circ}$ vertical field of view $\left(10^{\circ}\right.$ up and $18^{\circ}$ down). A Rockwell-Collins EPX5000 computer image generator created the out-the-window visual scene. The visual system equivalent time delay was $62 \mathrm{~ms}$ [17]. This was in line with the equivalent time delays of the motion system (Section III.C). The out-the-window visual system provided a visual scene in the clouds, without visual features that could be used to determine the attitude of the aircraft.

\section{Procedures}

Before the start of the experiment, pilots received an extensive briefing explaining the main purpose of the experiment and the general procedures. Pilots were told the motion settings would change between runs; however, no specifics were given about the different motion conditions. After the briefing, pilots filled out a short questionnaire with questions about their aircraft and simulator experience, after which they signed an informed consent form. After a safety walk-around and an explanation of the relevant simulator cab features, pilots were provided with an example run of the flight task by the experimenter without simulator motion.

Pilots performed seven runs under each of the four motion conditions (excluding training runs). Three training runs with the full-motion condition (FM) were performed at the start of the experiment for a total of 31 runs. After training, the first run in every block of four runs was always the full-motion condition. Pilots were told this was the baseline motion condition and the motion in the following three runs had to be rated against this one. In the remaining three runs the $\mathrm{HB}, \mathrm{HO}$, and $\mathrm{HA}$ motion conditions were presented in random order according to a balanced Latin square 
design. Each run lasted 200 seconds. The total run time of the experiment was three and a half hours, including briefing and break time. Two or three runs for each condition were performed in between 15-minute breaks. Participants were allowed to take longer brakes if requested.

Pilots provided two subjective motion ratings verbally per run, one for the heading capture task and one for the stall recovery task. An audio message prompted pilots to provide their rating. Pilots were asked to rate the motion during these tasks in reference to the full aircraft motion condition by providing a percentage between 0 and $100 \%$ at the end of each task. They were also asked to provide general comments about the motion after each run.

\section{Participants}

Eighteen general aviation pilots participated in the experiment. None of the pilots had experience with flying commercial transport aircraft. All pilots were comfortable with operating the joystick with their right hand. The average age of the participant pool was $26.8 \pm 5.5$ years $(\mu \pm \sigma)$. The average total flying hours was $507.1 \pm 606.4$ with $94.2 \pm 162.2$ hours in the last six months. Pilots had spent a total of $173.2 \pm 475.7$ hours in simulators, including personal-computer-based simulators, before taking part in the experiment. Pilots were compensated for their participation and provided informed consent.

\section{Dependent Measures}

Dependent measures were averaged over the seven measurement runs per condition for each pilot. The study considered two subjective motion ratings as dependent measures. Pilots rated the simulator motion in reference to the full motion condition FM verbally between 0\% (not similar to FM) and 100\% (equivalent to FM) after the heading changes, $r 1$, and the stall recovery, $r 2$.

Nine objective task performance parameters were considered as dependent measures [3]. The objective measures were calculated from the recorded simulator data which were sampled at $100 \mathrm{~Hz}$. All task performance parameters are indicated in Fig. 1. The gray areas in Fig. 1 show the time frame used to calculate a particular performance measure if applicable. Three measures determined performance during the heading-change task. During the heading changes, the root mean square (RMS) of the roll angle deviation from -15 or $15 \mathrm{deg}, R M S_{\phi h}$, was calculated from 25 to $40 \mathrm{~s}$ and 60 to $75 \mathrm{~s}$, respectively. The RMS of the pitch angle deviation from $8 \mathrm{deg}, R M S_{\theta}$, was calculated for the entire heading-change task (20-90 s). Finally, the mean error from the desired heading of 280 or $300 \mathrm{deg}$, $\bar{e}_{\phi}$, was captured using data at the end of each heading-change segment (50-55 s and 85-90 s).

Six objective performance measures related to the high-altitude stall task. The RMS of the roll angle, $R M S_{\phi s}$, applied to the entire stall task; that is, approach to stall and stall recovery. Altitude loss, $\Delta h, \operatorname{minimum~load~factor,~} n_{m i n}$, maximum load factor, $n_{\text {max }}$, and maximum calibrated airspeed, $V_{C A S-\max }$, applied to the stall recovery segment (Fig. 1). The number of additional stall warnings during the recovery, $N_{s}$, was determined starting $10 \mathrm{~s}$ after the initiation of the stall recovery from 150 to 200 s to allow sufficient time for the initial warning to go off.

\section{B. Hypotheses}

Several previous studies investigated pilot control behavior and performance in a stall recovery task under the baseline hexapod (HB), optimized hexapod (HO), and full motion (FM) conditions [4, 7, 9]. Hypotheses were formulated based on the findings of these studies and the notion that the adaptive hexapod motion condition (HA) provides motion similar to $\mathrm{HB}$ in the heading task and similar to HO in the stall task.

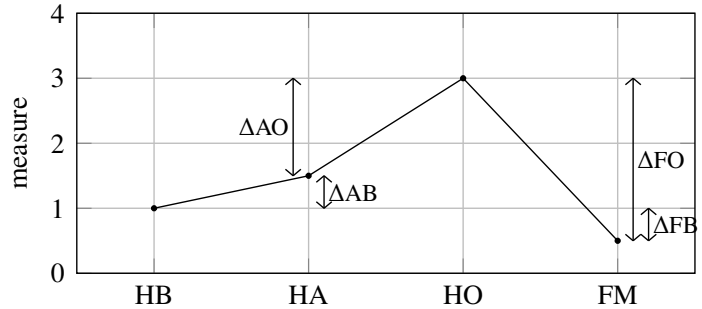

(a) Heading-task dependent measure.

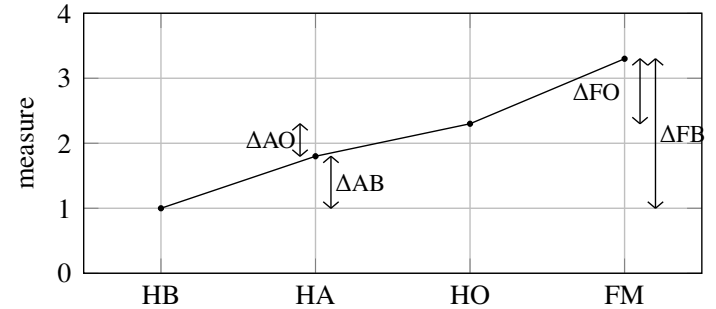

(b) Stall-task dependent measure.

Fig. 8 Visualization of hypotheses. 
Fig. 8 aids in defining the hypotheses. Fig. 8 a provides an example of a dependent measure in the heading task, and Fig. $8 \mathrm{a}$ an example of a measure in the stall task. Note that the data in these figures are not experimental results. The first two hypotheses compare the adaptive hexapod motion condition with the baseline hexapod and optimized hexapod motion conditions. Since the adaptive motion algorithm was designed to provide motion similar to HB in the heading task, when the aircraft is more stable, and motion similar to $\mathrm{HO}$ in the stall task, when he aircraft is more unstable, the following two hypotheses were defined:

$\mathrm{H} 1$ : Pilot motion ratings and task performance in the heading task $\left(r 1, R M S_{\phi h}, R M S_{\theta}\right.$, and $\left.\bar{e}_{\psi}\right)$ under HA motion were expected to be more similar to that under $\mathrm{HB}$ motion compared to $\mathrm{HO}$ motion. This means the difference between $\mathrm{HA}$ and $\mathrm{HB}(\triangle \mathrm{AB})$, and $\mathrm{HA}$ and $\mathrm{HO}(\triangle \mathrm{AO})$, is significantly different and $\triangle \mathrm{AB}$ is smaller than $\triangle \mathrm{AO}$ for those dependent measures (Fig. 8a).

$\mathrm{H} 2$ : Motion ratings and task performance in the stall task $\left(r 2, R M S_{\phi s}, \Delta h, n_{\min }, n_{\max }, V_{C A S}\right.$, and $\left.N_{s}\right)$ under HA motion were expected to be more similar to that under $\mathrm{HO}$ motion compared to HB motion. This hypothesis is true if $\triangle \mathrm{AB}$ and $\triangle \mathrm{AO}$ are significantly different and $\triangle \mathrm{AO}$ is smaller than $\triangle \mathrm{AB}$ for those dependent measures (Fig. 8b).

The last two hypotheses compare the baseline hexapod and optimized hexapod motion conditions with the full motion condition. HB simulated translational accelerations at the aircraft's c.g. albeit with reduced fidelity compared to FM. These translational accelerations might aid pilots in capturing the desired headings in the heading task. Translational accelerations of the c.g. were not present in the HO motion condition. However, HO motion provides higher fidelity motion cues related to aircraft attitude changes compared to HB. These characteristics of HB and HO resulted in the following hypotheses:

$\mathrm{H} 3$ : The average relative heading error $\bar{e}_{\psi}$ in the heading-capture task under $\mathrm{HB}$ motion as opposed to $\mathrm{HO}$ motion was expected to be more similar to that under FM motion, the baseline. This means the difference between FM and $\mathrm{HB}(\Delta \mathrm{FB})$, and $\mathrm{FM}$ and $\mathrm{HO}(\Delta \mathrm{FO})$, is significantly different and $\Delta \mathrm{FB}$ is smaller than $\Delta \mathrm{FO}$ for $\bar{e}_{\psi}$ (Fig. 8a). The opposite was expected to be true for the remaining measures in the heading task $\left(r 1, R M S_{\phi h}\right.$, and $\left.R M S_{\theta}\right)$, as these are all related to attitude changes.

H4: Dependent measures in the stall task $\left(r 2, R M S_{\phi s}, \Delta h, n_{\min }, n_{\max }, V_{C A S}\right.$, and $\left.N_{s}\right)$ under HO motion as opposed to $\mathrm{HB}$ motion were expected be more similar to that under FM motion. This means $\Delta \mathrm{FB}$ and $\Delta \mathrm{FO}$ are significantly different and $\Delta \mathrm{FO}$ is smaller than $\triangle \mathrm{FB}$ for those dependent measures (Fig. 8b).

In order to test the hypotheses, paired-samples t-tests were performed to detect significant differences between $\Delta \mathrm{FB}$ and $\triangle \mathrm{FO}$, and $\Delta \mathrm{HB}$ and $\Delta \mathrm{HO}$.

\section{Results}

This section presents the combined results of the 18 pilots that participated in the experiment. Error-bar plots present the continuous-interval dependent measures, with means and 95\% confidence intervals for each condition. The confidence intervals were corrected for between-subject variability. A bar plot presents the ordinal dependent measure, with the number of occurrences for each dependent measure level and the median for each experimental condition.

Paired-samples t-tests were used to determine whether the mean difference between $\triangle \mathrm{AB}$ and $\Delta \mathrm{AO}$, or $\Delta \mathrm{FB}$ and $\Delta \mathrm{FO}$, was statistically significantly different from zero (Fig. 8). For the t-test to produce reliable results, the data must meet two assumptions: 1) there should be no significant outliers in the differences between the two groups, and 2) the distribution of the differences between the two groups should be approximately normal. Outliers were identified using box plots and normality was checked using the Shapiro-Wilk test. Very few outliers were detected in any of the dependent measures and data were mostly normally distributed.

A Wilcoxon signed-rank test was used for the ordinal-level dependent measure. All statistical tests had a significance level of 0.05 . Table 1 provides a summary of the statistical test results for all the continuous repeated measures and the ordinal repeated measure (Section IV.A.5). In this table, $d f$ are the degrees of freedom, $t$ or $z$ is the test statistic, and $p$ is the probability of observing an effect. Cohen's $d$, was used as a measure for effect size. Effect sizes around 0.2, 0.5, and 0.8 reflect small, medium, and large effect sizes, respectively.

\section{A. Motion Ratings}

The motion ratings are provided in Fig. 9 by the black data. In general, the baseline hexapod motion was rated lowest and the optimized hexapod motion highest. The adaptive hexapod motion was rated in between $\mathrm{HB}$ and $\mathrm{HO}$ for both tasks. Note that FM was always rated $100 \%$; that is, pilots were always made aware this was the baseline which represented $100 \%$. 
Table 1 ANOVA results.

\begin{tabular}{|c|c|c|c|c|c|c|c|c|c|}
\hline & \multirow[t]{2}{*}{$\begin{array}{l}\text { Dependent } \\
\text { Measure }\end{array}$} & \multicolumn{4}{|c|}{$\begin{array}{c}\text { Difference Between } \\
\Delta \mathrm{AB} \text { and } \Delta \mathrm{AO}\end{array}$} & \multicolumn{4}{|c|}{$\begin{array}{c}\text { Difference Between } \\
\Delta \mathrm{FB} \text { and } \Delta \mathrm{FO}\end{array}$} \\
\hline & & $d f$ & $t, z$ & $p$ & $d$ & $d f$ & $t, z$ & $p$ & $d$ \\
\hline Motion & $r 1$ & 17 & -3.948 & 0.001 & -0.9306 & 17 & 3.948 & 0.001 & 0.9306 \\
\hline Ratings & $r 2$ & 17 & 2.082 & 0.053 & 0.4908 & 17 & 5.010 & $<0.0005$ & 1.1809 \\
\hline \multirow{3}{*}{$\begin{array}{l}\text { Heading } \\
\text { Task }\end{array}$} & $R M S_{\phi h}$ & 17 & -0.514 & 0.614 & -0.1213 & 17 & -1.241 & 0.231 & -0.2926 \\
\hline & $R M S_{\theta}$ & 17 & 0.463 & 0.649 & 0.1091 & 17 & -0.463 & 0.649 & -0.1091 \\
\hline & $\bar{e}_{\psi}$ & 17 & -2.676 & 0.016 & -0.6308 & 17 & 2.676 & 0.016 & 0.6308 \\
\hline \multirow{6}{*}{$\begin{array}{l}\text { Stall } \\
\text { Task }\end{array}$} & $R M S_{\phi s}$ & 17 & -0.631 & 0.537 & -0.1487 & 17 & -4.712 & $<0.0005$ & -1.1106 \\
\hline & $\Delta h$ & 17 & 0.880 & 0.391 & 0.2075 & 17 & -0.880 & 0.391 & -0.2075 \\
\hline & $n_{\min }$ & 17 & 0.999 & 0.332 & 0.2356 & 17 & 5.071 & $<0.0005$ & 1.1953 \\
\hline & $n_{\max }$ & 17 & 1.132 & 0.273 & 0.2667 & 17 & -2.082 & 0.053 & -0.4907 \\
\hline & $V_{C A S, \max }$ & 17 & 0.816 & 0.426 & 0.1922 & 17 & -0.816 & 0.426 & 0.1922 \\
\hline & $N_{s}$ & 17 & -1.000 & 0.317 & - & 17 & 1.000 & 0.317 & - \\
\hline
\end{tabular}

$=\quad$ significant $(p \leq 0.05)$

$=\quad$ suggestive $(0.05<p \leq 0.10)$

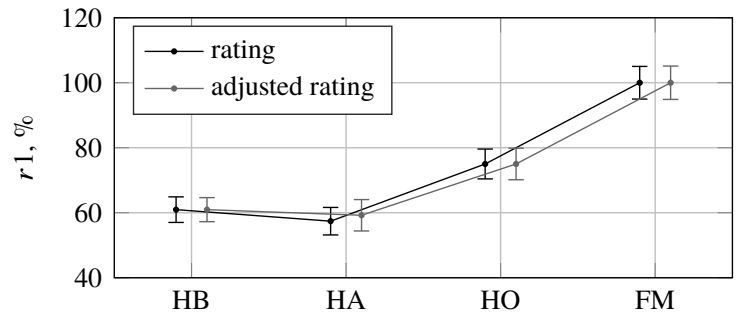

(a) Heading-task motion ratings.

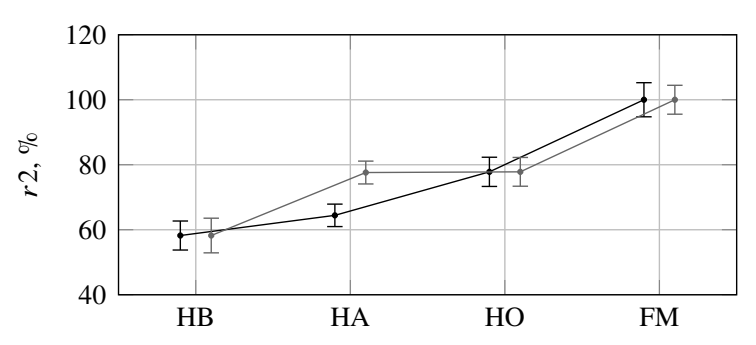

(b) Stall-task motion ratings.

Fig. 9 Motion ratings.

In the heading capture task (Fig. 9a), pilots rated the adaptive hexapod motion HA significantly more similar to the baseline hexapod motion HB compared to the optimized hexapod motion HO (Table 1). In addition, HO as opposed to $\mathrm{HB}$ was rated significantly more similar to FM. For the stall task (Fig. 9b), the difference between $\triangle \mathrm{AB}$ and $\triangle \mathrm{AO}$ was not significant; however, HA was rated more similar to HB compared to HO suggestively. Furthermore, $\mathrm{HO}$ as opposed to HB was rated significantly more similar to FM.

Ratings for the adaptive motion in the stall task were expected to be closer to the optimized hexapod motion. However, the adaptive motion varied between baseline and optimized hexapod motion during the scenario. To verify if this variation had an effect on pilots' perception of the motion overall, an adjusted motion rating was calculated using the average value of $C_{l_{p}}$ and taking into account the limits (Fig. 2). More information on the calculation of the adjusted ratings can be found in the appendix. The adjusted ratings are depicted in Fig. 9 by the gray data. Note that only the adjusted ratings for the adaptive motion are different compared to the original ratings.

For the heading task, the adjusted rating for the adaptive motion is similar to the unadjusted rating. This is to be expected as $C_{l_{p}}$ stays around the lower limit used for the motion parameter calculations during this task. However, for the stall task, the adjust rating for the adaptive motion is higher compared to the original rating and closer to the rating for the optimized hexapod motion as expected. These adjusted data serve as a possible explanation for why pilots rated HA closer to HB as opposed to $\mathrm{HO}$ in the stall task, even though HA provided motion more similar to HO near the stall point. No statistical test was performed on the adjusted ratings as it can't be verified if this adjustment is fair. 


\section{B. Task Performance}

Task performance for the heading task is provided in Fig. 10. The RMS of the roll and pitch attitudes is provided in Figs. 10a and 10b, respectively. Values for these measures were very similar for all hexapod motion conditions. Pilots were able to better keep the desired roll and pitch angles under FM; that is, the RMS values were lower. $R M S_{\phi h}$ and $R M S_{\theta}$ in HA were not significantly closer to HB or HO (Table 1). In addition, roll and pitch RMS values under HB or $\mathrm{HO}$ were not significantly closer to those under FM. It was easier for pilots to keep the desired pitch attitude during the turns compared to the roll attitude as indicated by the lower RMS values.

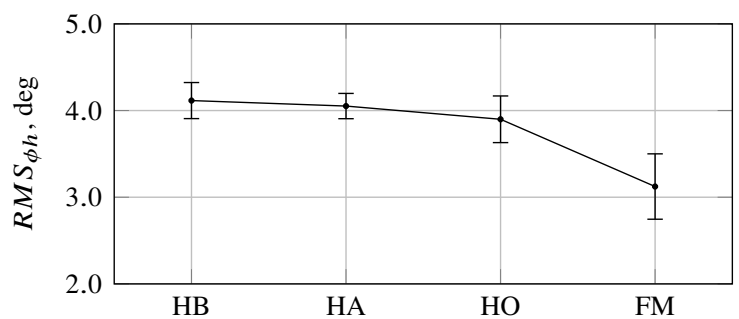

(a) RMS roll.

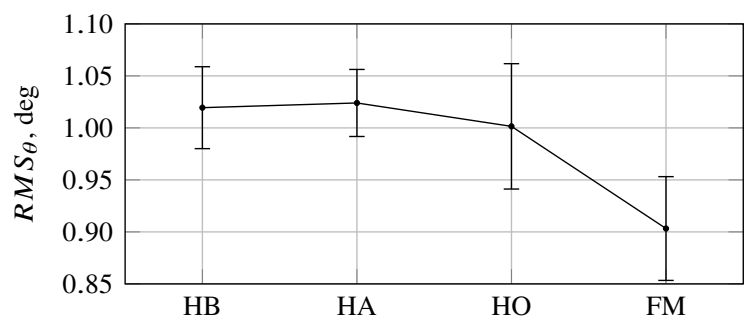

(b) RMS pitch.

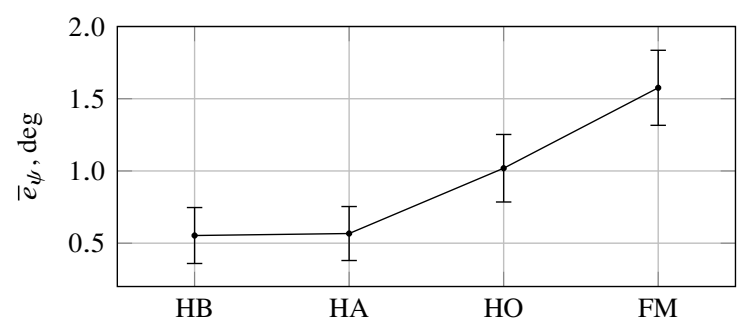

(c) Average relative heading error.

Fig. 10 Roll, pitch, and heading errors during the heading capture task.

Fig. 10c provides the average heading error after the heading captures during the heading task. Note that a negative value means pilots undershot the desired heading, while a positive value means they overshot the desired heading. The results indicate that pilots always overshot the desired heading. The desired heading was overshot the least for the baseline hexapod motion condition and the most for the full motion condition. The paired-samples t-test indicated that $\bar{e}_{\psi}$ under $\mathrm{HA}$ is significantly more similar to that under $\mathrm{HB}$ compared to HO. In addition, $\bar{e}_{\psi}$ under $\mathrm{HO}$ as opposed to $\mathrm{HB}$ was significantly more similar to FM. The later result is unexpected as the best heading-capture performance was expected under FM. This result could be due to the fact that the turbulence was also more noticeable under FM, negatively affecting heading-capture performance.

Fig. 11 provides the task performance for the stall task. The RMS of the roll angle is depicted in Fig. 11a. Values for $R M S_{\phi s}$ were very similar for all hexapod motion conditions (around $2.5 \mathrm{deg}$ ). Pilots were able to maintain wings level markedly better under FM motion. The overall trend in the roll RMS data is similar between the heading and stall tasks. However, note that is was easier to maintain the wings level during the stall task compared to keeping the desired roll angle during the turns in the heading task (comparing Figs. 10a and 11a). $R M S_{\phi s}$ under HA was not significantly more similar to that under either $\mathrm{HB}$ or $\mathrm{HO}$. However, $R M S_{\phi s}$ under $\mathrm{HO}$ as opposed to $\mathrm{HB}$ was significantly more similar to that under FM.

Fig. 11b depicts the altitude loss during the stall recovery. The average altitude loss was around 3,750 $\mathrm{ft}$ and was similar in all motion conditions. Altitude loss under HA or FM was not more similar to that under HB or HO (Table 1). The minimum and maximum load factors during the stall recovery are depicted in Figs. 11c and 11d, respectively. The minimum load factor, which occurred when pilots point the nose of the aircraft down to start the stall recovery, was lowest under $\mathrm{HB}$ and highest under HO and FM. $n_{\min }$ under HA was not significantly more similar to that under HB or HO. However, $n_{\min }$ under $\mathrm{HO}$ as opposed to HB was significantly more similar to that under FM. The maximum load factor occurred during the pull up in the stall recovery (Fig. 1). The maximum load factor appears to be slightly higher under HB and decreased going from HA, to HO and FM. The maximum load factor under HA was not significantly more similar to that under $\mathrm{HB}$ or HO. $n_{\max }$ under $\mathrm{HO}$ as opposed to $\mathrm{HB}$ was suggestively more similar to FM. Note that 


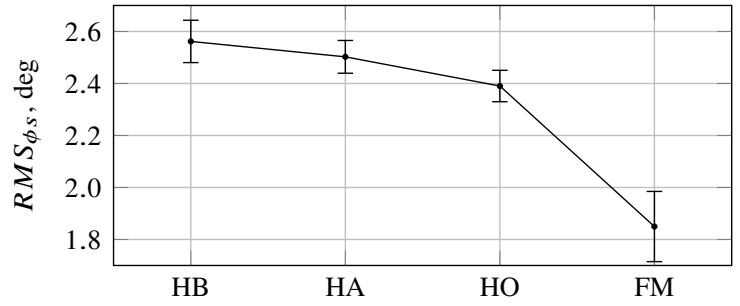

(a) RMS roll.

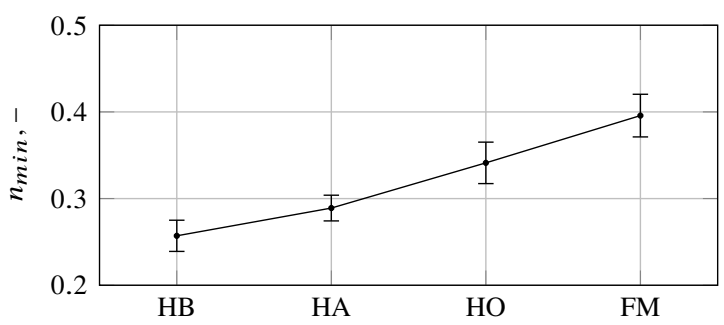

(c) Minimum load factor.

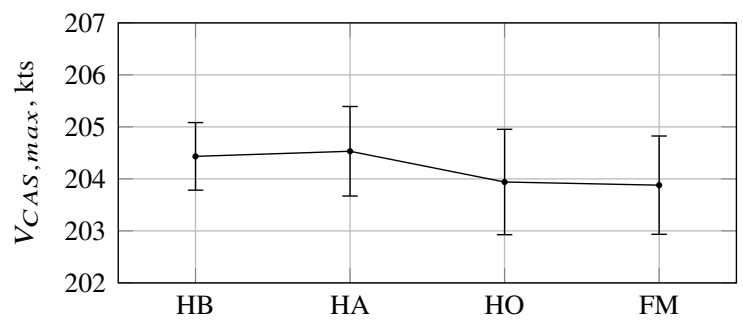

(e) Maximum airspeed.

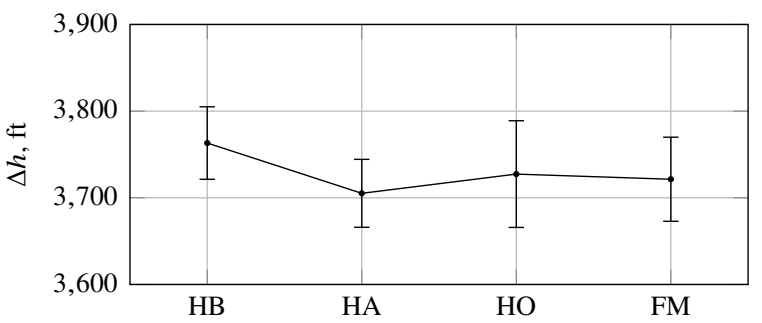

(b) Altitude loss.

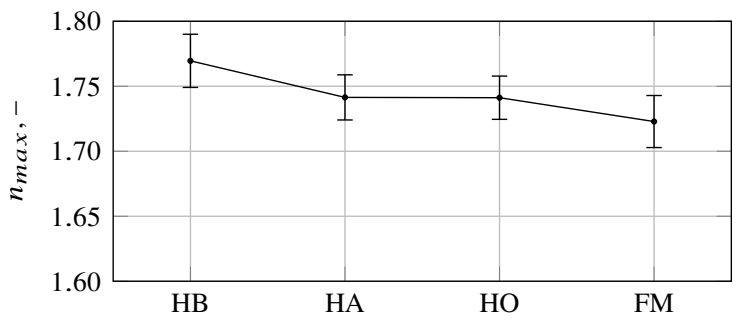

(d) Maximum load factor.

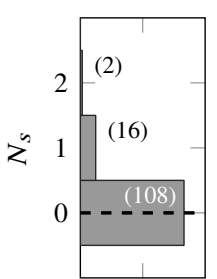

HB

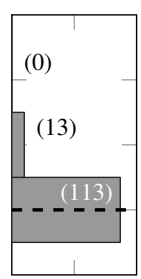

HA

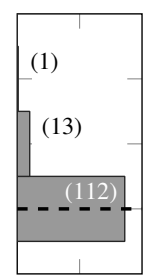

$\mathrm{HO}$

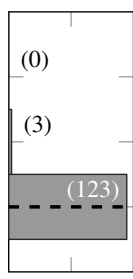

FM (f) Additional stall warnings.

Fig. 11 Performance measures during the stall recovery task.

the load factor always remained within normal transport aircraft limits $(-1.0<n<2.5)$.

The maximum calibrated airspeed during the stall recovery is depicted in Fig. 11e. The average maximum calibrated airspeed was around $204 \mathrm{kts}$ and was similar in all motion conditions. $V_{C A S, \max }$ under HA or FM was not significantly more similar to that under HB or HO. Finally, the number of additional stall warnings in the stall recovery are depicted in the bar plot of Fig. 11f. The results indicate more occurrences of secondary stall warnings under hexapod motion compared to full motion. The highest number of secondary stall warnings was found for HB, and the lowest number for FM. The medians of the data are zero for each motion condition. $N_{s s}$ under HA or FM was not significantly more similar to that under $\mathrm{HB}$ or $\mathrm{HO}$.

\section{Discussion}

An optimized hexapod motion cueing strategy for stall recovery training was evaluated in previous studies in the VMS and B747 simulators at NASA Ames Research Center [4, 7-9]. This optimized motion cueing strategy prioritizes translational accelerations as a result of rotations with respect to the c.g. over translational accelerations of the c.g., allowing for higher fidelity of the motion cues related to attitude changes [21]. The previous studies showed that pilot control behavior and performance in a stall task under the optimized motion was more similar to that under real aircraft motion compared to the baseline motion currently provided by most commercial transport simulators. However, this might not be the case for tasks where translational accelerations of the c.g. provide important cues for pilots, such as a heading- or altitude-capture task. This paper developed and evaluated an adaptive motion cueing algorithm based on aircraft stability that provided motion more similar to the baseline hexapod motion when the aircraft was in stable flight or more similar to the optimized hexapod motion when the aircraft was in unstable flight, such as a stall. An experiment 
was conducted on the VMS in which 18 general aviation pilots conducted a heading-capture task and stall recovery task consecutively under the baseline, optimized, and adaptive hexapod motion conditions, in addition to a full-motion condition, the baseline.

Paired-samples t-tests were used to detect if motion ratings and task performance under the adaptive hexapod motion, HA, were more similar to the baseline hexapod, HB, or optimized hexapod, HO, motion conditions in both tasks. In the heading-capture task, the motion rating $r 1$ and average relative heading error $\bar{e}_{\psi}$ under HA motion were both significantly more similar to those under HB motion compared to HO motion. The remaining two heading-capture-task performance measures, the RMS of the roll and pitch angles, $R M S_{\phi h}$ and $R M S_{\theta}$, were similar under all hexapod motion conditions and hence under HA not significantly more similar to either $\mathrm{HB}$ or $\mathrm{HO}$. We can therefore conclude that pilot motion ratings and task performance under $\mathrm{HA}$ were more similar to $\mathrm{HB}$ compared to $\mathrm{HO}$ in the heading-capture task, and hypothesis $\mathrm{H} 1$ can therefore be accepted (Section IV.B).

For the stall task, the motion rating $r 2$ under HA was suggestively more similar to $\mathrm{HB}$ as opposed to $\mathrm{HO}$, an unexpected result. However, when adjusting the motion rating by taking into account the fact that motion cues in HA varied between $\mathrm{HB}$ and $\mathrm{HO}$, it appeared to be more similar to HO. The adjustment used the average value of $C_{l_{p}}$ and its limits used for the motion parameter calculations (see appendix). It is not possible to determine if this adjustment is accurate as it assumes that pilots relied on their perception of the motion over the entire duration of the stall task and that their motion rating was linearly related to the strength of the motion. The stall-task performance measures, RMS of the roll angle $R M S_{\phi s}$, altitude loss $\Delta h$, minimum load factor $n_{\min }$, maximum load factor $n_{\max }$, maximum calibrated airspeed $V_{C A S \text {, max }}$, and number of additional stall warnings $N_{s}$, were all not significantly more similar to either HB or HO. Fig. 11 shows that task performance under HA was right in between performance under HB and HO. Therefore, hypothesis $\mathrm{H} 2$ can not be accepted; that is, motion ratings and task performance under HA were not more similar to HO compared to HB. This might be due to the fact that the adaptive motion is not more similar to the optimized motion for the entire duration of the stall task. A similar adjustment as the one used for the motion rating $r 2$ could be used to adjust the performance measures in the stall task; however, this was not attempted because of the concerns about its accuracy.

Paired-samples t-tests were also used to detect if motion ratings and task performance under $\mathrm{HB}$ or $\mathrm{HO}$ motion were significantly more similar to that under FM in both tasks. For the heading-capture task, all dependent measures under $\mathrm{HO}$ as opposed to HB were expected to be closer to FM, except for the average relative heading error $\bar{e}_{\psi}$ as performance in capturing a heading was expected to benefit from the translational accelerations of the c.g. simulated in HB and FM, but not in HO. Both $r 1$ and $\bar{e}_{\psi}$ under $\mathrm{HO}$ as opposed to $\mathrm{HB}$ were significantly more similar to those under FM. $R M S_{\phi h}$ and $R M S_{\theta}$ under $\mathrm{HB}$ or $\mathrm{HO}$ were equally similar to FM. As $\bar{e}_{\psi}$ under $\mathrm{HO}$ as opposed to $\mathrm{HB}$ was more similar to FM, hypothesis $\mathrm{H} 3$ could not be accepted. Note that heading-capture performance was worst under FM motion even though this motion condition had the highest fidelity translational aircraft c.g. motion. A possible explanation for this could be the presence of larger motion disturbances under FM as a result of the simulated turbulence.

In the stall task, the motion rating $r 2$, the RMS of the roll attitude $R M S_{\phi s}$, and the minimum load factor $n_{\min }$ under $\mathrm{HO}$ as opposed to HB were all significantly more similar to those under FM. The maximum load factor $n_{\max }$ under $\mathrm{HO}$ as opposed to HB was suggestively more similar to that under FM. The remaining dependent measures related to the stall task ( $\triangle h, V_{C A S, \max }$, and $\left.N_{s}\right)$ were similar between all motion conditions. This allows us to conclude that the motion rating and task performance in stall task under $\mathrm{HO}$ as apposed to $\mathrm{HB}$ motion was more similar to those under FM, and accept hypothesis $\mathrm{H} 4$.

Figs. 10 and 11 indicate that pilot performance in both tasks is similar under all three hexapod motion conditions. Limited differences in performance might have been present due to the structured nature of the task, for example, due to the use of desired attitude indicators on the PFD. More effects could be observed in less structured tasks. In addition, pilot control behavior and performance might have been more variable under the adaptive motion as the motion is also dependent on pilots' control inputs. Furthermore, the experiment used general aviation pilots which might not have been as comfortable with controlling the commercial transport dynamics. Finally, task performance is usually not an effective measure to determine the effects of different simulator motion configurations as pilots adapt their control behavior to different motion cues. Human operator models characterizing manual control behavior with different parameters are usually a better way to investigate the effects of different motion conditions.

Current adaptive motion algorithms in simulators have adaptive motion parameters based on the location of the simulator in the motion space. As different components of the total aircraft motion might be more important dependent on the task or flight regime [4], an adaptive motion algorithm with motion parameters dependent on aircraft parameters or task variables might be more appropriate. This paper proposed an adaptive motion algorithm dependent on the aircraft roll damping coefficient $C_{l_{p}}$ with motion varying between baseline hexapod motion which might be more suitable for tasks in the stable flight regime and optimized hexapod motion for tasks in the unstable flight regime such as 
a stall recovery. The roll damping coefficient is a convenient parameter as it is normally readily available in lookup tables that are part of the aircraft dynamic model [9]. However, $C_{l_{p}}$ was not available in lookup tables for the GTM model used and had to be estimated using a recursive least squares algorithm. This solution proved to be effective. However, the purpose of this paper was not to provide the ultimate solution for an adaptive motion algorithm based on aircraft parameters, but to what extent such an approach to adaptive motion is feasible. This paper showed that the presented solution to adaptive motion is promising and can be implemented in a straightforward way. More research is required to test the adaptive motion algorithm in different tasks. In addition, different solutions for adaptive motion based on aircraft parameters or tasks variables should be tested.

\section{Conclusion}

This study used the Vertical Motion Simulator at NASA Ames Research Center and a transport aircraft model to determine the feasibility of an adaptive hexapod simulator motion algorithm based on aircraft roll stability. Eighteen general aviation pilots flew a heading-capture task and a stall task consecutively under four motion configurations: baseline hexapod, adaptive hexapod, optimized hexapod, and full motion. The adaptive motion was more similar to the baseline hexapod motion in the heading-capture task when the aircraft was more stable, and more similar to the optimized hexapod motion in the stall task when the aircraft was more unstable. Pilot motion ratings and task performance in the heading-capture task under the adaptive hexapod motion were more similar to baseline hexapod motion compared to optimized hexapod motion. However, motion ratings and task performance in the stall task under the adaptive motion were not significantly more similar to the optimized hexapod motion compared to baseline hexapod motion. This might be due to the fact that the adaptive motion varied between the baseline and optimized hexapod motion conditions over the course of the stall task. Motion ratings and overall task performance under optimized hexapod motion as opposed to baseline hexapod motion were always more similar to the full motion condition. This was not expected for the heading-capture performance as the translational aircraft c.g. motion in both the baseline hexapod and full motion conditions was thought to assist in capturing the heading. This paper showed that adaptive motion based on aircraft stability is feasible and can be implemented in a straightforward way. More research is required to test the adaptive motion algorithm in different tasks.

\section{Appendix}

\section{A. Adjusted Motion Ratings}

The adjusted motion rating for the heading task $r 1_{\text {adj }}$ was calculated according to:

$$
r 1_{a d j}=\frac{C_{l_{p} u l}-C_{l_{p} l l}}{C_{l_{p} u l}-\bar{C}_{l_{p}}} r 1
$$

where $C_{l_{p} u l}$ is the upper limit used for the motion parameter calculations and $C_{l_{p} l l}$ is the lower limit (Fig. 2). $\bar{C}_{l_{p}}$ is the average value for $C_{l_{p}}$ during the heading task. The adjusted rating for the stall task $r 2_{a d j}$ was calculated in a similar way. Although not shown in this paper, Other dependent measure could be adjusted in a similar way.

\section{B. Simulator Motion Filters}

A more general overview of the VMS motion logic and motion system are given in [17]. More details about the motion filters and parameter values for each motion configuration are provided here.

The VMS motion logic is completely linear in its operating envelope; that is, the only nonlinear element is the motion limiting near the boundaries of the envelope. The motion configurations of the experiment were tuned such that these motion boundaries were never reached. Motion filtering was performed in the inertial reference frame. Aircraft model accelerations were transformed from the aircraft body reference frame to the inertial reference frame using small-angle approximations. The VMS and hexapod motion algorithms used gains and second-order high-pass washout filters to attenuate accelerations in the translational and rotational degrees of freedom:

$$
H_{m}=K_{m} \frac{s^{2}}{s^{2}+2 \zeta_{m} \omega_{m} s+\omega_{m}^{2}}
$$




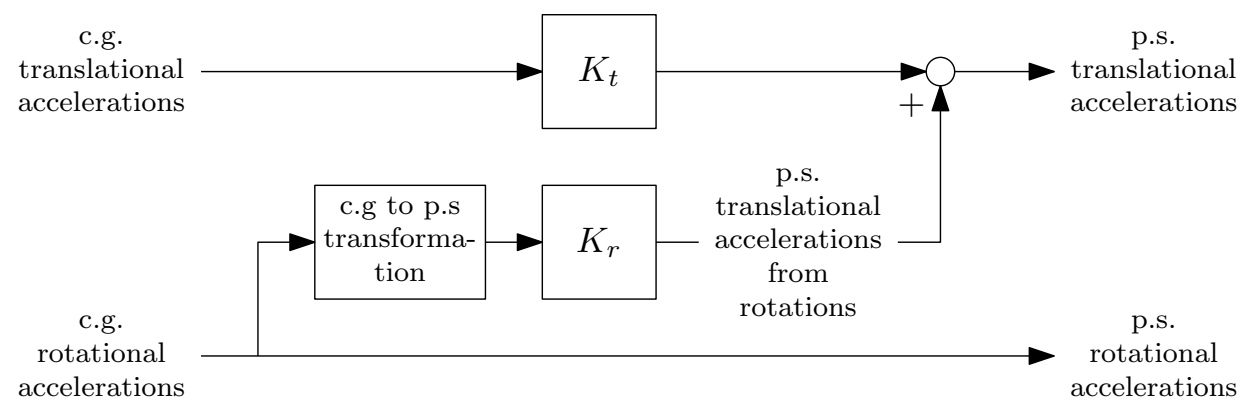

Fig. 12 Transformation from center of gravity to pilot station accelerations.

where $s$ is the Laplace variable, $K_{m}$ is the motion gain, $\zeta_{m}$ is the washout damping ratio, and $\omega_{m}$ is the washout break frequency. Both the motion gains $K_{m}$ and the break frequencies $\omega_{m}$ were adaptive in the adaptive hexapod motion condition HA.

The different motion conditions in the experiment not only differed in motion logic parameter settings, but also in the type of translational accelerations simulated. Translational accelerations at the pilot station are a combination of translational accelerations of the aircraft's center of gravity (c.g.) and translational accelerations as a result of the pilot station (p.s.) rotating with respect to the center of gravity (Fig. 12). Gains on each of the translational acceleration components $\left(K_{t}\right.$ and $\left.K_{r}\right)$ allowed for a different weighting of each component [4]. $K_{t}$ and $K_{r}$ were also adaptive in the adaptive hexapod motion condition HA.

Table 2 provides the motion gains and washout break frequencies of all four motion configurations. All damping ratios were set to 0.707 .

Table 2 Motion filter parameters.

\begin{tabular}{cccccc}
\hline \hline & & \multicolumn{3}{c}{ Motion Configuration } \\
\cline { 3 - 6 } & Motion & Baseline & Optimized & Adaptive & Full \\
& Parameter & Hexapod (HB) & Hexapod (HO) & Hexapod (HA) & Motion (FM) \\
\hline Motion Component & $K_{t}$ & 1.00 & 0.00 & $1.00-0.00$ & 1.00 \\
Gains & $K_{r}$ & 1.00 & 0.75 & $1.00-0.75$ & 1.00 \\
\hline Motion Filter & $K_{m x}$ & 0.50 & 0.50 & $0.50-0.51$ & 0.50 \\
Gains & $K_{m y}$ & 0.60 & 0.70 & $0.60-0.70$ & 1.00 \\
& $K_{m z}$ & 0.50 & 0.70 & $0.50-0.70$ & 0.80 \\
& $K_{m p}$ & 0.60 & 1.00 & $0.60-1.00$ & 1.00 \\
Motion Filter & $K_{m q}$ & 0.60 & 0.90 & $0.60-0.90$ & 0.90 \\
Break Frequencies & $K_{m r}$ & 0.60 & 1.00 & $0.60-1.00$ & 1.00 \\
\hline & $\omega_{m x}$ & 2.00 & 1.00 & $2.00-1.00$ & 0.80 \\
& $\omega_{m y}$ & 2.00 & 1.00 & $2.00-1.00$ & 0.20 \\
& $\omega_{m p}$ & 2.75 & 1.00 & $2.75-1.00$ & 1.00 \\
& $\omega_{m q}$ & 0.75 & 0.40 & $0.75-0.30$ & 0.20 \\
\hline \hline
\end{tabular}

\section{Acknowledgments}

The authors thank everyone at NASA Ames SimLabs who contributed to developing and running the experiment. We also thank the eighteen pilots who participated in the experiment. Finally, we would also like to thank Dr. Thomas Lombaerts for his help and support during the initial setup of the experiment. This work was supported by NASA's 
Technologies for Airplane State Awareness project for which Dr. Gautam Shah was the technical program coordinator.

\section{References}

[1] Flight Simulation Training Device Qualification Standards for Extended Envelope and Adverse Weather Event Training Tasks, 14 CFR PART 60, US Department of Transportation, Federal Aviation Administration, 2016.

[2] Schroeder, J. A., Bürki-Cohen, J., Shikany, D. A., Gingras, D. R., and Desrochers, P., “An Evaluation of Several Stall Models for Commercial Transport Training," Proceedings of the AIAA Modeling and Simulation Technologies Conference, National Harbor (MD), 2014. doi:10.2514/6.2014-1002.

[3] Grant, P. R., Moszczynski, G. J., and Schroeder, J. A., "Post-stall Flight Model Fidelity Effects on Full Stall Recovery Training," 2018 Modeling and Simulation Technologies Conference, American Institute of Aeronautics and Astronautics, 2018. doi:10.2514/6.2018-2937.

[4] Zaal, P. M. T., and Zavala, M. A., "Effects of Different Heave Motion Components on Pilot Pitch Control Behavior," AIAA Modeling and Simulation Technologies Conference, American Institute of Aeronautics and Astronautics (AIAA), 2016. doi:10.2514/6.2016-3371.

[5] Zaal, P. M. T., and Sweet, B. T., "The Challenges of Measuring Transfer of Stall Recovery Training," Proceedings of the 2014 IEEE International Conference on Systems, Man, and Cybernetics, San Diego, CA, 2014, pp. 3138-3143. doi: 10.1109/SMC.2014.6974410.

[6] Zaal, P. M. T., Popovici, A., and Zavala, M. A., "Effects of False Tilt Cues on the Training of Manual Roll Control Skills," Proceedings of the AIAA Modeling and Simulation Technologies Conference, Kissimmee, Florida FL, 2015. doi: 10.2514/6.2015-0655.

[7] Zaal, P. M. T., and Mobertz, X. R. I., "Effects of Motion Cues on the Training of Multi-Axis Manual Control Skills," AIAA Modeling and Simulation Technologies Conference, AIAA, Denver, Colorado, 2017. doi:10.2514/6.2017-3473.

[8] Popovici, A., Zaal, P., and Pieters, M. A., "Time-Varying Manual Control Identification in a Stall Recovery Task under Different Simulator Motion Conditions," 2018 Modeling and Simulation Technologies Conference, American Institute of Aeronautics and Astronautics, 2018. doi:10.2514/6.2018-2936.

[9] Zaal, P. M. T., Chung, W. W., Carpenter, D. M., Cunningham, K., and Shah, G. H., "Verification of a Motion Cueing Strategy for Stall Recovery Training in a Commercial Transport Simulator," AIAA Modeling and Simulation Technologies Conference, American Institute of Aeronautics and Astronautics (AIAA), 2019. doi:10.2514/6.2019-0426.

[10] Zaichik, L. E., Yashin, Y. P., Desyatnik, P. A., and Smaili, H., "Some Aspects of Upset Recovering Simulation On Hexapod Simulators," Proceedings of the AIAA Modeling and Simulation Technologies Conference, Minneapolis (MN), 2012. doi:10.2514/6.2012-4949.

[11] Nooij, S. A. E., Wentink, M., Smaili, H., Zaichik, L., and Groen, E. L., "Motion Simulation of Transport Aircraft in Extended Envelopes: Test Pilot Assessment," Journal of Guidance, Control, and Dynamics, 2016, pp. 1-13. doi:10.2514/1.g001790.

[12] Ko, S. F., and Grant, P. R., "Development and Testing of an Adaptive Motion Drive Algorithm for Upset Recovery Training," Proceedings of the AIAA Modeling and Simulation Technologies Conference, Minneapolis (MN), 2012. doi:10.2514/6.2012-4947.

[13] Hueschen, R. M., "Development of the Transport Class Model (TCM) Aircraft Simulation From a Sub-Scale Generic Transport Model (GTM) Simulation," Technical Report NASA/TM-2011-217169, NASA Langley Research Center, Hampton, Virginia, 2011. URL https://ntrs.nasa.gov/search.jsp?R=20110014509.

[14] Hyde, D. C., Brown, F. R., Shweyk, K. M., and Shah, G. H., "Advanced Modeling and Uncertainty Quantification for Flight Dynamics (VSST1-7) Interim Results and Challenges," AIAA Atmospheric Flight Mechanics Conference, American Institute of Aeronautics and Astronautics, 2014. doi:10.2514/6.2014-0035.

[15] Lombaerts, T., Schuet, S., Stepanyan, V., Kaneshige, J., Hardy, G., Shish, K. H., and Robinson, P., "Piloted Simulator Evaluation Results of Flight Physics Based Stall Recovery Guidance," 2018 AIAA Guidance, Navigation, and Control Conference, American Institute of Aeronautics and Astronautics, 2018. doi:10.2514/6.2018-0383.

[16] Campbell, S., Kaneshige, J., Nguyen, N., and Krishnakumar, K., "An Adaptive Control Simulation Study using Pilot Handling Qualities Evaluations," AIAA Guidance, Navigation, and Control Conference, American Institute of Aeronautics and Astronautics, 2010. doi:10.2514/6.2010-8013. 
[17] Beard, S. D., Reardon, S. E., Tobias, E. L., and Aponso, B. L., "Simulation System Optimization for Rotorcraft Research on the Vertical Motion Simulator," Proceedings of the AIAA Modeling and Simulation Technologies Conference, Minneapolis (MN), 2012. doi:10.2514/6.2012-4634.

[18] Parrish, R. V., Dieudonne, J. E., Bowles, R. L., and Martin, D. J., Jr., "Coordinated Adaptive Washout for Motion Simulators," Journal of Aircraft, Vol. 12, No. 1, 1975, pp. 44-50.

[19] Hosman, R. J. A. W., and Advani, S. K., “Are Criteria for Motion Cueing and Time Delays Possible? Part 2.” Proceedings of the AIAA Modeling and Simulation Technologies Conference, Boston (MA), 2013. doi:10.2514/6.2013-4833.

[20] Zaal, P. M. T., Schroeder, J. A., and Chung, W. W., "Refinement of Objective Motion Cueing Criteria Based on Three Flight Tasks," AIAA Modeling and Simulation Technologies Conference, American Institute of Aeronautics and Astronautics (AIAA), 2017. doi:10.2514/6.2017-1081.

[21] Zaal, P. M. T., Pool, D. M., de Bruin, J., Mulder, M., and van Paassen, M. M., "Use of Pitch and Heave Motion Cues in a Pitch Control Task," Journal of Guidance, Control, and Dynamics, Vol. 32, No. 2, 2009, pp. 366-377. doi:10.2514/1.39953. 